\title{
Concerns about modelling of the EDGES data
}

ARISING From J. D. Bowman, A. E. E. Rogers, R. A. Monsalve, T. J. Mozdzen \& N. Mahesh Nature 555, 67-70 (2018); https://doi.org/10.1038/ nature25792

It is predicted ${ }^{1}$ that the spectrum of radio emission from the whole sky should show a dip arising from the action of the light from the first stars on the hydrogen atoms in the surrounding gas, which causes the $21-\mathrm{cm}$ line to appear in absorption against the cosmic microwave background. Bowman et al. ${ }^{2}$ identified a broad flat-bottomed absorption profile centred at $78 \mathrm{MHz}$, which could be this feature, although the depth of the profile is much larger than expected. We have examined the modelling process they used and find that their data implies unphysical parameters for the foreground emission and also that their solution is not unique, in the sense that we found other simple formulations for the signal that are different in shape but that also fit their data. We argue that this calls into question the interpretation of these data as an unambiguous detection of the cosmological $21-\mathrm{cm}$ absorption signature. There is a Reply to this Comment by Bowman, J. D. et al. Nature 564, https://doi.org/10.1038/s41586-0180797-4 (2018).

Bowman et al. ${ }^{2}$ describe a 'physically motivated' foreground model containing three parameters describing synchrotron emission (magnitude, spectral index and the 'running' of the index) and two for ionospheric emission and absorption. They used a linearized version of this model to perform fits with and without the $21-\mathrm{cm}$ feature. Using this model and the data that they released, we obtained essentially identical results, but we note that accommodating the proposed absorption profile requires a change in the foreground model that
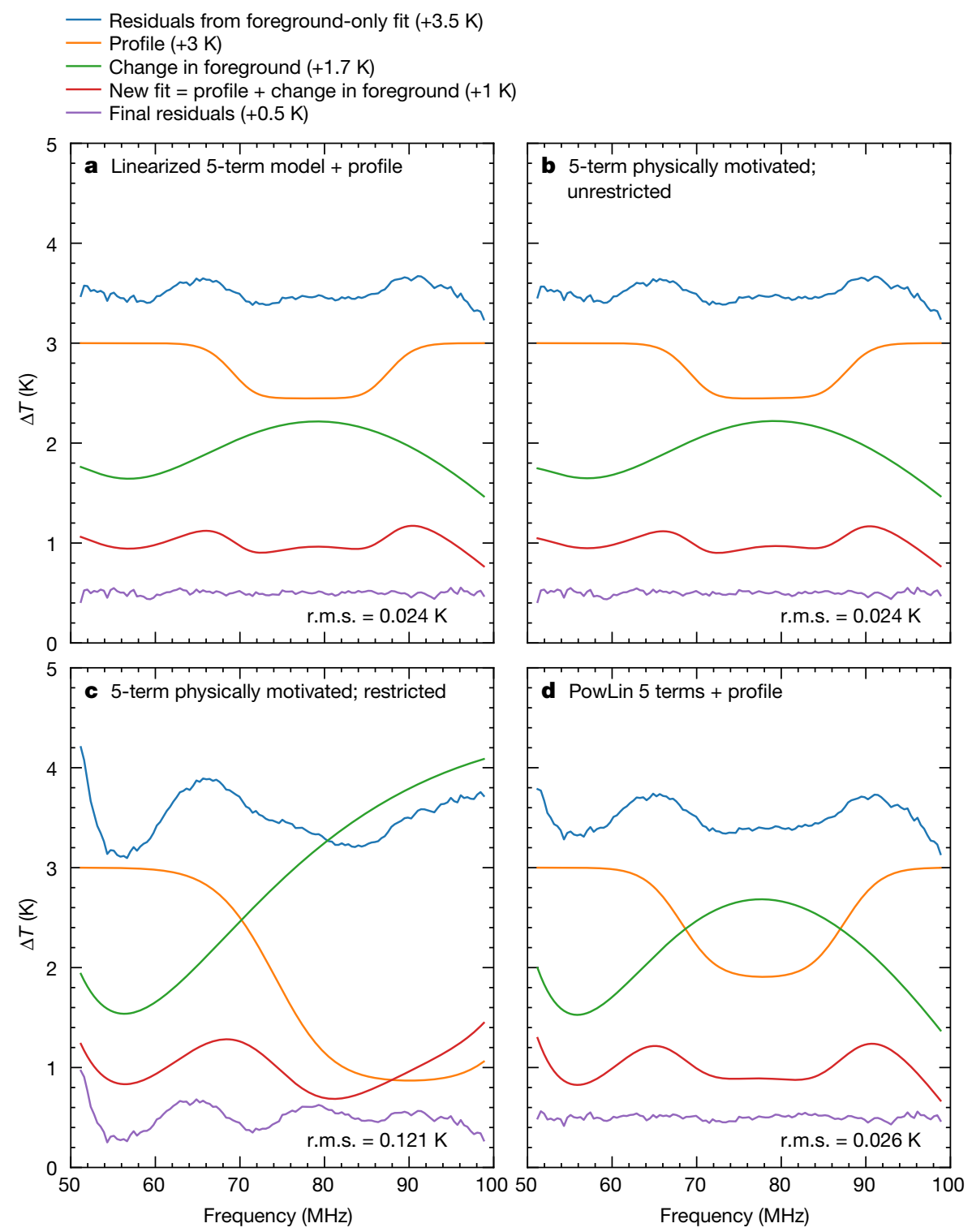

Fig. 1 Fits to the EDGES data. a, With the foregrounds described by the linearized function used by Bowman et al. ${ }^{2}$. b, Using the physically motivated nonlinear function for the foregrounds and no restrictions on the parameters. $\mathbf{c}$, The same as b but with the range of parameters limited to physically plausible values. d, Using the PowLin model, which consists of a power law with index given by a polynomial in frequency, $\nu$. The top line in each panel shows the residuals when a fit is made using the foreground model only. The bottom line is the residual when the fit is run again including the profile with the functional form given by Bowman et al. ${ }^{2}$. The intermediate lines show the shape of the profile found, the change in the foreground model needed to accommodate this and the sum of these two, which is also equal to the difference between the initial and final residuals. The curves have been offset vertically for readability. (r.m.s., root mean square.) 

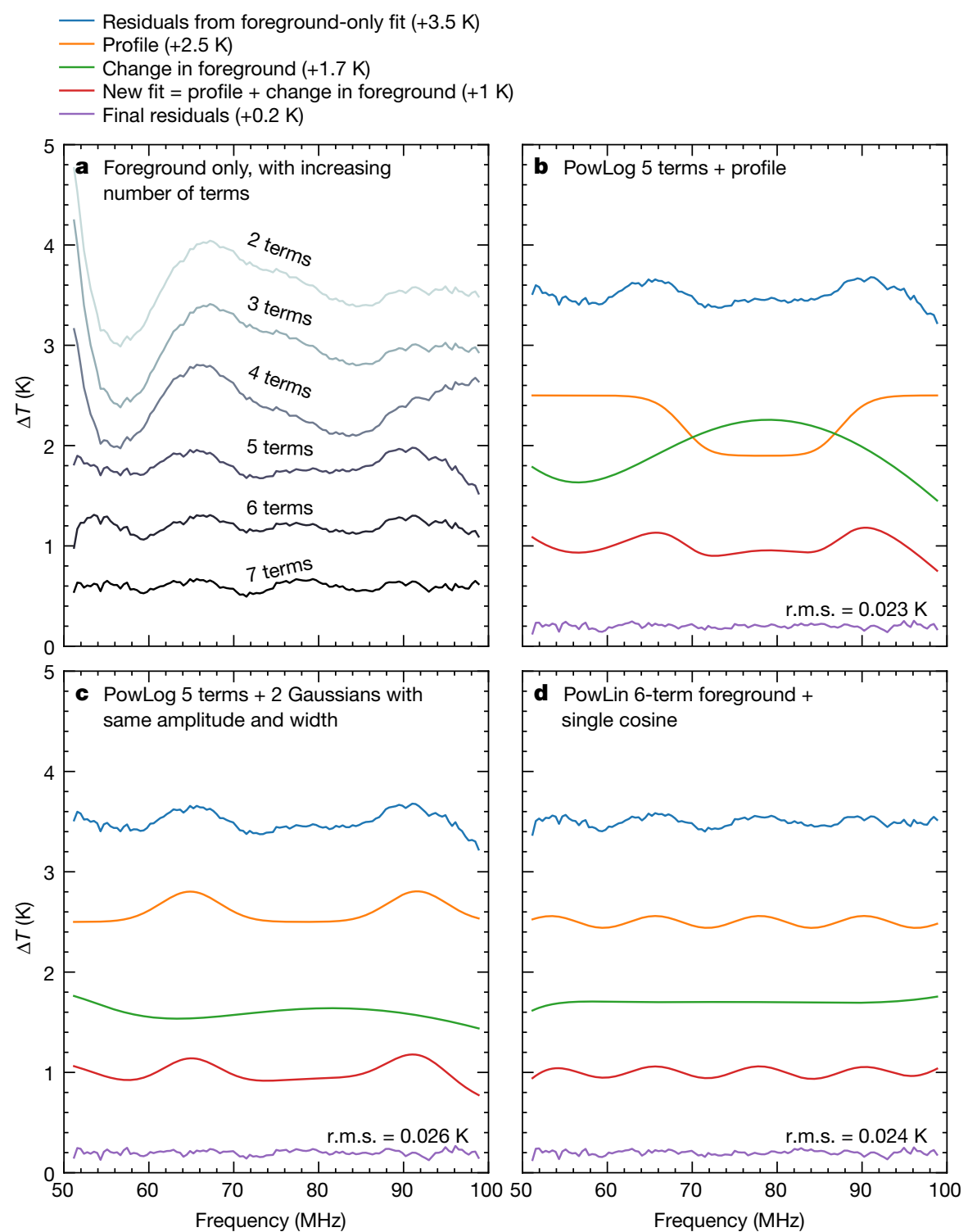

Fig. 2 Further illustrations of the fitting process. a, The residuals when the ionospheric parameters are set to $\tau_{0}=0.014$ and $T_{\mathrm{e}}=800 \mathrm{~K}$ and the PowLog model-a power law with index given by a polynomial in $\log (\nu)$-is fitted with increasing numbers of terms. b. Showing how the Bowman et al. ${ }^{2}$ profile with four signal parameters can provide a good fit by making use of the freedom provided by five foreground parameters.

is much larger than the initial residuals; see Fig. 1a. We also found that the parameters describing the foregrounds have unphysical values; for example, the parameter associated with brightness temperature of the ionospheric emission exceeds $10^{4} \mathrm{~K}$, while that for the astronomical foreground brightness has a large negative value. Full details of the functions fitted, together with the values found for the parameters, are given in the Supplementary Information to this Comment.

To gain further insight we fitted the full non-linear expression, taking into account the linkage of the emission and absorption by the ionosphere via the temperature of the electrons, $T_{\mathrm{e}}$. The values found for the optical depth of the ionosphere $\tau_{\text {ion }}$ and for $T_{\mathrm{e}}$ are both negative, which is clearly unphysical. We constrained these parameters to physically plausible values ${ }^{3}, \tau_{\text {ion }}>0.005$ at $75 \mathrm{MHz}$ and $200<T_{\mathrm{e}}<2,000 \mathrm{~K}$, and we restricted the centre frequency of the absorption profile to lie

c, d, Alternative nine-parameter fits. c, The same model as b but with two Gaussian features of equal width and amplitude in place of the Bowman et al. ${ }^{2}$ profile. d, The PowLin model with six terms and, instead of the profile, a single cosine function, which has a fitted amplitude of about $0.06 \mathrm{~K}$. The different curves have again been offset vertically for readability.

between 60 and $90 \mathrm{MHz}$. The results obtained with and without these restrictions on the parameters are shown in Fig. 1b, c. Without the restrictions we obtained essentially the same profile as Bowman et al. ${ }^{2}$ and the fit is good. (We describe the fit as 'good' whenever the root mean square of the residuals is below $0.03 \mathrm{~K}$.) With the restrictions the fit is poor; the centre of the profile has moved to the upper limit and its depth has increased to about $2 \mathrm{~K}$.

We then explored cases where the ionospheric opacity and temperature are held fixed at reasonable values but higher-order terms are added to the foreground model, using several different formulations. We found that at least five free foreground parameters, in addition to the four absorption profile parameters, were always needed to obtain a good fit. The parameters found for the profile changed substantially when different formulations for the foreground were used. Figure 1d shows an example of this, where a good fit was obtained with an 
amplitude of about $1.1 \mathrm{~K}$ for the absorption feature, which is even larger than that found by Bowman et al. ${ }^{2}$.

The residuals found when fitting with successively higher numbers of terms in the foreground model are shown in Fig. 2a. We note that at no stage in this process does a distinct absorption-line feature appear. Instead, a broad oscillatory feature is present when two, three or four terms are used and it is only the addition of the fifth term that produces a large reduction in the residuals. Although it can be argued that higher-order terms are needed to represent the synchrotron foreground accurately, this is not the behaviour expected ${ }^{4}$. In particular, the relatively large value required for the fifth term is not consistent with what is known ${ }^{5-8}$ about the spectrum of the foreground emission in the range $25-400 \mathrm{MHz}$. Adding higher-order foreground terms has simply moved the problem of unphysical parameter values from the ionosphere to the foreground.

It seems possible that the unphysical values are due to residual systematic errors in the data, perhaps arising in the correction for the frequency-dependent beam shape, but if that is the case then it is not clear that model formulations chosen to suit the astronomical foregrounds are the correct way of removing such effects and there is also no clear basis for deciding how many terms should be included. A general concern is that nine parameters are being fitted to data that span $50 \mathrm{MHz}$ and contain very little real structure with periods shorter than about $10 \mathrm{MHz}$, so neighbouring data points in the spectrum are strongly correlated. This means that the number of truly independent data points may not be much larger than the number of parameters being fitted.

We next demonstrated that the profile found by Bowman et al. ${ }^{2}$ is not a unique solution. The top lines of Fig. 1a, b show the residuals after subtracting a five-parameter foreground fit. As already noted, the ionosphere parameters have unphysical values in those cases. The fourth line down in Fig. 2a shows that very similar residuals are obtained by assuming reasonable fixed values for the ionosphere and fitting a five-parameter power law. The residuals do not, however, show an absorption profile but instead show two peaks at around $65 \mathrm{MHz}$ and $90 \mathrm{MHz}$. Although one can obtain a good fit using the Bowman et al. ${ }^{2}$ profile (Fig. 2b), a good fit can also be achieved with two Gaussian emission features of equal height and width (Fig. 2c). With more terms in the foreground model (that is, the bottom lines in Fig. 2a), the residuals take the form of undulations with a period of around $12.5 \mathrm{MHz}$. We found that a satisfactory fit can then be obtained with just a sine wave, as shown in Fig. $2 d$. In both of these models the total number of free parameters is again nine.

We also found that, with the $12.5 \mathrm{MHz}$ sine wave removed, a good fit was obtained with five foreground parameters and a broad Gaussian absorption profile and that there is then a large covariance between the foreground and signal components. This suggests that these undulations may be what causes the fitting process used by Bowman et al. ${ }^{2}$ to produce a profile with a flattened bottom. Since the proposed $21-\mathrm{cm}$ absorption profile does not match theoretical expectations in either shape or amplitude, it is not clear why it should be preferred to the other forms of signal explored here or to the many more that can be found in the degenerate space between signal and foreground model. Therefore, although our analysis does not prove that the feature identified by Bowman et al. ${ }^{2}$ is absent from their data, we believe the issues that we have raised are such that the evidence for its presence falls well short of the level required to invoke new physics for its explanation.

G.K. acknowledges support from ERC Advanced Grant 320596 'The Emergence of Structure During the Epoch of Reionization'. P.D.M. and E.P. acknowledge support from Senior Kavli Institute Fellowships at the University of Cambridge. P.D.M. also acknowledges support from The Netherlands Organization For Scientific Research (NWO) VIDI grant (dossier 639.042.730).

\section{Methods}

We used a least-squares fitting for testing the models presented in the text and the Supplementary Information. In addition, we derived posterior distributions of model parameters by implementing a likelihood function into the multi-nested sampler Polychord ${ }^{9,10}$.

\section{Data availability}

For all our analyses we used the 'Data for Figure 1 of Bowman et al. (2018)' in the EDGES Data Release, which is available at http://oco.lab.asu.edu/edges/edgesdata-release/.

\section{Richard Hills $^{1 *}$, Girish Kulkarni ${ }^{2,3,7}$, P. Daniel Meerburg ${ }^{2,3,4,5,6}$ \&} Ewald Puchwein ${ }^{2,3,8}$

${ }^{1}$ Cavendish Laboratory, University of Cambridge, Cambridge, UK. ${ }^{2}$ Institute of Astronomy, University of Cambridge, Cambridge, UK. ${ }^{3}$ Kavli Institute for Cosmology, University of Cambridge, Cambridge, UK. ${ }^{4}$ Department of Applied Mathematics and Theoretical Physics, University of Cambridge, Cambridge, UK.

${ }^{5}$ Kapteyn Astronomical Institute, University of Groningen, Groningen, The Netherlands.

${ }^{6}$ Van Swinderen Institute for Particle Physics and Gravity, University of Groningen, Groningen,

The Netherlands.

7Present address: Department of Theoretical Physics, Tata Institute of Fundamental Research, Mumbai, India.

8Present address: Leibniz Institute for Astrophysics, Potsdam, Germany. *e-mail: richard@mrao.cam.ac.uk

Received: 3 May 2018; Accepted: 15 October 2018; Published online 19 December 2018.

1. Pritchard, J. R. \& Loeb, A. $21 \mathrm{~cm}$ cosmology in the 21st century. Rep. Prog. Phys. 75, 086901 (2012).

2. Bowman, J. D., Rogers, A. E. E., Monsalve, R. A., Mozdzen, T. J. \& Mahesh, N. An absorption profile centred at 78 megahertz in the sky-averaged spectrum. Nature 555, 67-70 (2018).

3. Rogers, A. E. E., Bowman, J. D., Vierinen, J., Monsalve, R. \& Mozdzen, T. Radiometric measurements of electron temperature and opacity of ionospheric perturbations. Radio Sci. 50, 130-137 (2015).

4. Bernardi, G., McQuinn, M. \& Greenhill, L. J. Foreground model and antenna calibration errors in the measurement of the sky-averaged $\lambda 21 \mathrm{~cm}$ signal at $z \sim 20$. Astrophys. J. 799, 90 (2015).

5. Bridle, A. H. The spectrum of the radio background between 13 and $404 \mathrm{MHz}$. Mon. Not. R. Astron. Soc. 136, 219-240 (1967).

6. de Oliveira-Costa, A. et al. A model of diffuse Galactic radio emission from $10 \mathrm{MHz}$ to $100 \mathrm{GHz}$. Mon. Not. R. Astron. Soc. 388, 247-260 (2008).

7. Mozdzen, T. J., Bowman, J. D., Monsalve, R. A. \& Rogers, A. E. E. Improved measurement of the spectral index of the diffuse radio background between 90 and $190 \mathrm{MHz}$. Mon. Not. R. Astron. Soc. 464, 4995-5002 (2017).

8. Zheng, $\mathrm{H}$. et al. An improved model of diffuse galactic radio emission from $10 \mathrm{MHz}$ to $5 \mathrm{THz}$. Mon. Not. R. Astron. Soc. 464, 3486-3497 (2017).

9. Handley, W. J., Hobson, M. P. \& Lasenby, A. N. POLYCHORD: nested sampling for cosmology. Mon. Not. R. Astron. Soc. 450, L61-L65 (2015).

10. Handley, W. J., Hobson, M. P. \& Lasenby, A. N. POLYCHORD: nested sampling for cosmology. Mon. Not. R. Astron. Soc. 453, 4385-4399 (2015).

Author contributions E.P. initiated this study by pointing out the importance of the role of the foreground parameters in the claimed detection of an absorption profile. All authors participated in the detailed analysis and the writing of the Comment.

\section{Competing interests Declared none.}

Additional information

Supplementary information is available for this paper at https://doi.org/ 10.1038/s41586-018-0796-5.

Reprints and permissions information is available at http://www.nature.com/ reprints.

Correspondence and requests for materials should be addressed to R.H.

https://doi.org/10.1038/s41586-018-0796-5 


\section{Reply to Hills et al.}

REPLYING TO R. Hills et al. Nature 564, https://doi.org/10.1038/s41586-018-0796-5 (2018)

In our Letter ${ }^{1}$, the foreground models account for a combination of astronomical foregrounds, ionospheric effects and any residual calibration effects. We obtained best-fit parameters that absorb a degenerate combination of these effects from unconstrained fits to the models. In the accompanying Comment ${ }^{2}$, the concerns of Hills et al. arise primarily because they failed to recover physical values for two ionosphere parameters in a foreground model with three additional non-ionosphere parameters.

Ionosphere parameters are covariant with the amplitude and spectral index of the astronomical foreground. Small errors in these astronomical parameters, as well as residual effects from calibration, could bias the recovered ionosphere parameters. In ref. ${ }^{3}$, we calculated an overall systematic uncertainty of \pm 0.02 on the spectral index measured by the high-band instrument, including beam correction uncertainty. We found a similar uncertainty for the low-band instrument. Errors of this level could yield deviations from the true spectrum with amplitudes and shapes comparable to those of the expected ionospheric contribution. For these reasons, we did not intend to extract ionospheric information from the measurements presented. In a previous study ${ }^{4}$, we extracted information about ionospheric variability from EDGES highband data. We limited that analysis to differencing spectra acquired at the same local sidereal time on different nights in order to reduce the covariance with the astronomical foregrounds and mitigate any systematic effects, before fitting an ionosphere model to the differential spectra. Extracting absolute ionospheric information directly from the measured spectra would require a separate, in-depth study.

Measuring physical foreground properties requires the absolute temperature calibration of the spectrum, whereas identifying a $21-\mathrm{cm}$ profile embedded in the foreground requires only relative calibration between channels in the spectrum. It is possible to recover a $21-\mathrm{cm}$ feature without accurately measuring the physical foreground properties. Most global 21-cm constraints have come from this regime ${ }^{5-9}$. In EDGES we do aim to measure a fully absolutely calibrated spectrum. Although in our Methods section we acknowledged potential residual calibration effects, we reported tests to show that any such effects are not consistent with the reported profile. We therefore concluded that the signal is astronomical.

Hills et al. ${ }^{2}$ found that several alternative models for the foreground and signal can be fitted to the data. We broadly agree, but a general absorption profile remains the most justified a priori choice of signal model because we have disfavoured the instrument as the source of the structure and there is no known physical expectation for other shapes in either the foreground or 21-cm signal, whereas an absorption is expected. We have data that exclude some of the alternative signal models proposed by Hills et al. ${ }^{2}$ and plan to publish those results in the near future.

When using our polynomial foreground model over the full band (51-99 MHz), rather than over only the sub-band for which we used it (approximately 63-99 MHz), Hills et al. ${ }^{2}$ recovered best-fit profiles that are not consistent with our reported properties. We have shown using simulations ${ }^{10}$ that this outcome is consistent with the expected performance of that model. Therefore, their choice to use it over the full band was not justified. Other foreground models perform better than the polynomial model across the full band, including the linear physically motivated model that we used.

Judd D. Bowman ${ }^{1 *}$, Alan E. E. Rogers ${ }^{2}$, Raul A. Monsalve ${ }^{1,3,4,5,6}$, Thomas J. Mozdzen ${ }^{1}$ \& Nivedita Mahesh ${ }^{1}$

${ }^{1}$ School of Earth and Space Exploration, Arizona State University, Tempe, AZ, USA.

${ }^{2}$ Haystack Observatory, Massachusetts Institute of Technology, Westford, MA, USA.

${ }^{3}$ Department of Physics, McGill University, Montréal, Quebec, Canada. ${ }^{4}$ McGill Space Institute, McGill University, Montréal, Quebec, Canada. ${ }^{5}$ Center for Astrophysics and Space Astronomy, University of Colorado, Boulder, CO, USA.

${ }^{6}$ Facultad de Ingeniería, Universidad Católica de la Santísima Concepción, Concepción, Chile.

*e-mail: judd.bowman@asu.edu

Published online 19 December 2018.

1. Bowman, J. D., Rogers, A. E. E., Monsalve, R. A., Mozdzen, T. J. \& Mahesh, N. An absorption profile centred at 78 megahertz in the sky-averaged spectrum. Nature 555, 67-70 (2018).

2. Hills, R., Kulkarni, G., Meerburg, P. D. \& Puchwein, E. Concerns about modelling of the EDGES data. Nature 564, http://doi.org/10.1038/s41586-018-0797-4 (2018).

3. Mozdzen, T. J., Bowman, J. D., Monsalve, R. A. \& Rogers, A. E. E. Improved measurement of the spectral index of the diffuse radio background between 90 and $190 \mathrm{MHz}$. Mon. Not. R. Astron. Soc. 464, 4995-5002 (2017).

4. Rogers, A. E. E., Bowman, J. D., Vierinen, J., Monsalve, R. \& Mozdzen, T. Radiometric measurements of electron temperature and opacity of ionospheric perturbations. Radio Sci. 50, 130-137 (2015).

5. Bowman, J. D. \& Rogers, A. E. E. Lower limit of $\Delta z>0.06$ for the duration of the reionization epoch. Nature 468, 796-798 (2010).

6. Bernardi, G. et al. Bayesian constraints on the global $21-\mathrm{cm}$ signal from the cosmic dawn. Mon. Not. R. Astron. Soc. 461, 2847-2855 (2016).

7. Singh, S. et al. First results on the epoch of reionization from first light with SARAS 2. Astrophys. J. Lett. 845, L12 (2017)

8. Singh, S. et al. SARAS 2 constraints on global $21 \mathrm{~cm}$ signals from the epoch of reionization. Astrophys. J. Lett. 858, 54 (2018).

9. Price, D. C. et al. Design and characterization of the Large-aperture Experiment to Detect the Dark Age (LEDA) radiometer systems. Mon. Not. R. Astron. Soc. 478, 4193-4213 (2018).

10. Bowman, J. Foreground model selection for signal parameter estimation. EDGES report 122, http://loco.lab.asu.edu/loco-memos/edges_reports/ report122.pdf (2018).

Author contributions J.D.B., R.A.M. and A.E.E.R. contributed equally to this Reply. N.M. and T.J.M. provided input and approved the final response.

Competing interests Declared none.

Additional information

Reprints and permissions information is available at http://www.nature.com/ reprints.

Correspondence and requests for materials should be addressed to J.D.B.

https://doi.org/10.1038/s41586-018-0797-4 